\author{
J. Matias ${ }^{1}$, Â. Gaspar ${ }^{2}$, L. M. Borrego ${ }^{2,3}$, S. Piedade 2 , G. Pires², C. Arêde 2 , \\ G. SAMPAIO 2 , M. Morais-Almeida ${ }^{2}$
}

\title{
Tree nuts anaphylaxis in preschool age children
}

\author{
${ }^{1}$ Pediatric Department, Garcia de Orta Hospital, Almada, Portugal \\ ${ }^{2}$ Immunoallergy Department, CUF Descobertas Hospital, Lisbon, Portugal \\ ${ }^{3}$ CEDOC, Chronic Diseases Research Center, Immunology, NOVA Medical School, Faculdade de Ciências Médicas, \\ Lisbon, Portugal
}

\section{KEY WORDS \\ Anaphylaxis; children; food allergy; preschool age; tree nuts.}

\section{Corresponding author \\ Ângela Gaspar \\ Immunoallergy Department \\ CUF Descobertas Hospital \\ Mário Botas Street \\ 1998-018 Lisboa, Portugal \\ E-mail: angela.gaspar@sapo.pt}

\section{Doi}

10.23822/EurAnnACI.1764-1489.128

\begin{abstract}
Summary
Introduction. The incidence of food-induced anaphylaxis (FIA) is increasing in young children. Although the commonest culprits are cow's milk and egg, FIA to tree nuts (TNs) have been increasing. Objective. Characterization of children referred to our allergy department due to TNs-induced anaphylaxis (TNs-FIA) during preschool age. Materials and methods. We have retrospectively included 25 children with clinical history of preschool TNs-FIA, proven by allergological work-up. TNs sensitization was assessed by skin prick tests andlor specific IgE. Results. The mean age of the first anaphylactic episode was $3.1 \pm 1.2$ years. The majority (92\%) had an allergic disease ( $52 \%$ asthma). The implicated TNs were cashew (11 children), walnut (8), pine nut (5), hazelnut (2) and almond (1). The reaction occurred after the first known ingestion in 68\%. In 92\%, symptoms appeared within 30 minutes after exposure. The most frequent clinical symptoms were mucocutaneous (96\%), respiratory (80\%) and gastrointestinal (52\%). Twenty-one children were admitted to the emergency department, although only $48 \%$ were treated with epinephrine. An underneath IgE-mediated mechanism was proven in all cases. Immunologic cross-reactivity with other TNs was identified in 84\%, and with peanut in 36\%. Overall, in our center, TNs-FIA represents $18 \%$ of all causes of FIA. Conclusions. In preschool age children with TNs-FIA, cashew and walnut were the commonest implicated nuts. Most reactions occurred briefly after exposure to minimal amounts of TNs, demonstrating the high potency of these allergens. About one-third also had peanut sensitization. Potentially life-threatening TNs allergy can occur early in childhood and adequate management should be undertaken.
\end{abstract}

\section{Introduction}

Food allergy usually develops early in life and affects up to $10 \%$ of children (1-4). Its incidence and severity has been increasing worldwide, especially in preschool children (3-8). In this age group, the two most common food allergens are cow's milk and hen's egg (9-13). Nevertheless, severe allergic reactions to tree nuts (TNs) have been increasingly reported worldwide, both in raw form and within processed foods (7,8,14-16).

TNs are defined as a dry fruit composed of an inedible hard shell and a seed. The TNs that account for most allergic reactions are walnut, hazelnut, almond, pistachio, cashew, pecan nut, mac- adamia, brazil nut and pine nut $(16,17)$. Although botanically unrelated, TNs and peanut (that belongs to the botanical family of Leguminosae) share many allergenic similarities (17).

Overall, TNs allergy affects 0.5 to $3 \%$ of the population $(17,18)$, representing 11 to $40 \%$ of cases of food-induced anaphylaxis (FIA) $(13,16,19)$. However, its prevalence differs according to the geographical region and dietary patterns $(3,16-18,20)$. In the United States, walnut is the most commonly reported culprit of TNs allergy, followed by cashew and almond $(17,20)$. In Europe, hazelnut allergy is the most prevalent (17). The exact TNs allergy prevalence in Portugal is unknown. 
TNs allergy typically presents by the age of two to three years old, often after the first known ingestion (16-18). Symptoms are typically immunoglobulin $\mathrm{E}$ ( $\mathrm{IgE}$ ) mediated and arise from a massive mediator release from mast cells and basophils degranulation, usually appearing few seconds to two hours after the contact with the allergen $(3,7,21)$. The pathogenesis of non-IgE-mediated tree nuts allergy is less clear. Despite their underlying mechanism, non-IgE-mediated reactions are clinically indistinguishable and have similar acute management (21). The nature of the symptoms is often related to the age of the child, to the amount of the exposure (ingestion of large quantities is generally responsible for more rapid and pronounced reactions), and also to the ingested TN (cashew is associated with more severe reactions and airway narrowing) $(18,20)$. In infants up to two years old, the most common symptoms are cutaneous and gastrointestinal, and in preschool children, cutaneous, gastrointestinal, and respiratory symptoms are the most prominent. Severe symptoms as throat tightness are more frequent in older children and adolescents. Cardiovascular symptoms are less frequent than in adults $(7,9,12)$.

Risk factors for the development of TNs allergy include severe atopic eczema, egg allergy and the presence of family or personal history of allergic disease $(20,21)$. The concomitant presence of asthma and eczema, a history of allergic reactions to extremely small amounts of food and a history of a previous food-induced anaphylactic reaction, are associated with more serious manifestations $(16,18,20)$. TNs allergic reactions can be severe and account, together with peanut, for a high proportion of fatal FIA (up 70 to $90 \%)(15-17,20)$. The majority of fatal accidental reactions occur in adolescents and young adults (median age 27 years old) $(18,20)$.

Children with TNs allergy have a significantly increased risk of co-sensitization or co-allergy to other nuts $(16,18,20)$. The sensitization rate to TNs is high, reaching up to $86 \%{ }^{16}$ Certain specific TN allergies appear to coexist more commonly, such as cashew with pistachio or walnut with pecan $(16,18)$. Co-allergy between TNs and peanut ranges between $20 \%$ and $68 \%$ $(16,18,20)$.

The aim of this study was to perform the characterization of children referred to our allergy department due to FIA related to TNs ingestion during preschool age.

\section{Materials and methods}

We have retrospectively evaluated the medical reports of a group of children with FIA after TNs ingestion during preschool age, proven by allergological work-up, followed at the Immunoallergy department of CUF Descobertas Hospital (Lisbon, Portugal). The evaluation of these children was included in a systematic reporting of anaphylaxis which was implemented in our allergy outpatient department over eight years (from January
2011 to December 2018). All allergists of the department were invited to participate and a meeting was organized in order to promote the voluntary notification of cases of FIA.

The diagnosis of TNs induced-anaphylaxis was assumed when "at least one episode of severe systemic reaction" (as defined by the international consensus) $(7,13,22)$ occurred after the ingestion of TNs (described by the patient or caregiver) to which a confirmed sensitization has been proven. TNs were defined as walnut, almond, pistachio, cashew, pecan, hazelnut, macadamia, Brazil nut and pine nut.

The sensitization to TNs was assessed by in vivo skin prick tests (commercial extracts, Roxall-Aristegui ${ }^{\circledR}$, Bilbao, Spain) and/or in vitro assays of serum specific IgE (ImmunoCAP, Thermo Fisher Scientific ${ }^{\circledR}$, Waltham, Massachusetts, USA). Skin prick tests were performed by an allergist using standard methodology; the result was considered positive if the mean wheal diameter was 3 $\mathrm{mm}$ or greater, with negative control $(0.9 \%$ saline $)$ and positive control (histamine $10 \mathrm{mg} / \mathrm{mL}$ ). Regarding in vitro tests, results were considered positive if specific IgE was $0.35 \mathrm{kU} / \mathrm{L}$ or greater. The sensitization to peanut and aeroallergens was also assessed. In addition to this allergological diagnostic work-up, the authors collected demographic and clinical data: age, gender, family history of allergic disease, personal history of allergic disease and atopy (defined as positive skin prick test for at least one aeroallergen), date of the first anaphylactic reaction and a detailed description of the clinical manifestations (mucocutaneous, respiratory, gastrointestinal and cardiovascular). Moreover, the following information has also been collected: implicated TNs, elapsed time between exposure and the onset of symptoms, estimated amount of ingested TNs, place of anaphylaxis occurrence, attendance to the emergency department, description of the performed treatment (including information about the use of epinephrine) and management after the anaphylactic event (including information about prescription of epinephrine auto-injectors).

A descriptive statistical analysis was performed using SPSS for Windows version $20.0^{\circledR}$.

\section{Results}

Twenty-five children with a confirmed diagnosis of TNs-induced anaphylaxis during preschool age were included. The current mean age was 8.5 years ( $\mathrm{SD} \pm 3.8$ years) and $17(68 \%)$ were male.

Almost all (92\%) children had a personal history of other allergic disease: $20(80 \%)$ allergic rhinitis or rhinoconjunctivitis, $14(56 \%)$ atopic dermatitis, $13(52 \%)$ asthma, $4(16 \%)$ other food allergy ( 3 with cow's milk allergy, 2 with egg allergy, 1 with lamb allergy and 1 with peach allergy) and 1 (4\%) eosinophilic esophagitis. Eighteen children (72\%) were atopic (14 sensitized to house dust-mites, 7 to pollens and 4 to pets). A family history of allergy was identified in 21 children (84\%); four had family 
history of food allergy ( 2 with allergy to crustaceans, 1 to walnut and 1 to cow's milk).

The mean age of the first anaphylactic reaction to TNs was 3.1 years $(S D \pm 1.2$ years; minimum age of 14 months, maximum age of five years). In 16 children (64\%) the first episode occurred in the first three years of life.

The TNs involved in the allergic reaction were: cashew in 11 children, walnut in 8, pine nut in 5, hazelnut in 2 and almond in 1 child. There was one case of walnut and hazelnut-induced anaphylaxis and one case with cashew and almond.

In 19 children (76\%), the anaphylactic reaction was triggered by the ingestion of extremely small amounts of the TN involved. Ten children ingested a vestigial content, nine children ingested fragments or a single $\mathrm{TN}$, five children ingested two or more tree TNs (maximum of three units) and one child ingested an unknown quantity.

The anaphylactic reaction occurred after the first known TN ingestion in 17 cases (68\%). Only one child had a known allergy to walnut, and the anaphylactic episode occurred after an accidental exposure during holidays.

Regarding the place where the allergic reaction occurred, in 18 children $(72 \%)$ the anaphylactic episode occurred at home, in 4 $(16 \%)$ at a restaurant, in $1(4 \%)$ at the beach and in $2(8 \%)$ on vacation at recreational sites.

Concerning the reported symptoms, 24 patients (96\%) had mucocutaneous symptoms (urticaria, angioedema, pruritus), 20 $(80 \%)$ respiratory symptoms (cough, wheezing, dyspnea, stridor), 13 (52\%) gastrointestinal symptoms (vomiting, diarrhea, abdominal pain) and three (12\%) cardiovascular symptoms (prostration). Four children (16\%) presented life-threatening glottis edema. There were no fatal events.

Considering the infant subgroup (children who had two or less than two years old at the time of the first anaphylactic episode), corresponding to ten children ( $40 \%$ of the studied sample), all (100\%) had mucocutaneous symptoms, eight (80\%) respiratory (including two cases of glottis edema), four (40\%) gastrointestinal and one (10\%) cardiovascular manifestations. In the older children subgroup (60\% of the studied sample), 14 (93\%) had mucocutaneous symptoms, $12(80 \%)$ respiratory (including two cases of glottis edema), $9(60 \%)$ gastrointestinal and 2 $(13 \%)$ cardiovascular manifestations. Due to the small sample size, the authors did not carry out a comparative analysis between the two subgroups.

In 23 children (92\%), symptoms appeared within the first 30 minutes after contact with the implicated allergen. In seventeen children $(68 \%)$ the reaction occurred in the first five minutes, in six (24\%) between five and thirty minutes and in two (8\%) more than 30 minutes after the TN ingestion. The maximum time elapsed between the TN intake and the anaphylactic reaction was two hours (in one girl, after the ingestion of cereals with walnut and hazelnut).
Twenty-one out of the 25 children (84\%), were admitted to the emergency department during this first anaphylactic episode. However, among patients who have been observed in the emergency department, only $10(48 \%)$ were treated with intramuscular epinephrine. We must emphasize that an auto-injector epinephrine was prescribed to all children in our allergy department.

In all cases, an underlying IgE mediated mechanism was proven, by positive skin prick test and/or positive specific $\mathrm{IgE}$ to the culprit TN. Immunologic cross-reactivity (positive skin testing and/or in vitro immunoassays for specific $\mathrm{IgE}$ ) with other TNs was confirmed in 21 children (84\%) and with peanut in $9(36 \%)$.

Overall, TNs induced-anaphylaxis represented $18 \%$ of all causes of FIA in our department, from a total of 277 cases of anaphylaxis related to food ingestion. Moreover, regarding the triggers of FIA in the pediatric age group ( $<18$ years), from a total of 158 patients, although some patients reacted to more than one food allergen, the most frequent implicated foods were: cow's milk in $51(32 \%)$; TNs in 34 (22\%); egg in $20(13 \%)$; fresh fruits in 16 (10\%), 9 to Rosacea fruits; peanut in $13(8 \%)$; shellfish in 10 (6\%), 7 to shrimp; fish in 7 (4\%); seeds in 4 (3\%), 3 to sesame seed and 1 to sunflower seed.

\section{Discussion}

FIA is an important health problem, with an increasing incidence in preschool age $(5,6,8,12)$. TNs allergy is becoming more frequent worldwide, particularly in young children (14$16,20)$. In our center, we found TNs as an important trigger of FIA ( $18 \%$ of all FIA reports). We stress out that among the causes of FIA in our pediatric patients, it represents the second cause of FIA (22\%), after cow's milk (32\%).

In our pediatric sample, cashew and walnut were the most implicated TNs in preschool anaphylaxis. These are also the most allergenic TNs in the United States $(17,21)$. However, hazelnut is the most commonly reported TN allergy in Europe $(17,21)$, although allergy to hazelnut is often seen in the context of pollen-fruit syndrome due to PR-10 proteins sensitization, which causes mainly oral allergy syndrome and usually no anaphylactic reactions.

In a recent study also performed in our country, TNs were found to be the main cause of FIA (19\%). In fact, in 62 patients observed in a food allergy outpatient department at Coimbra, walnut and hazelnut have been spotted has the most implicated TNs (19). It seems that the reported prevalence can vary significantly, according to the age range of the studied population and the geographical region.

Most of the anaphylactic reactions occurred with the first known ingestion of TNs, and briefly after exposure to minimal amounts of the implicated food. This clearly demonstrates the 
high potency of these allergens. Therefore, successful elimination diets should include systematic education of the patient and their caregivers about food allergen labelling, proper food preparation and the risk of cross-contamination and hidden food ingredients (3). We highlight that the caregivers of these children may not be aware of the eventual accidental exposures that may have occurred and may have contributed to the occurrence of sensitization. Therefore, the diagnosis of food allergy cannot be excluded even if the caregivers deny previous contact of the child with the implicated allergen.

Mucocutaneous symptoms were the most frequent manifestations in these children. Respiratory and gastrointestinal symptoms were also very frequent, as reported by other authors in preschool age $(7,23)$, being cardiovascular symptoms more uncommon than in adulthood. There were no fatalities, but four children presented life-threatening glottis edema. Indeed, TNs can cause severe allergic reactions.

In all studied children, an underlying IgE mediated mechanism was proven (by in vivo or in vitro tests). In fact, the most common form of FIA is IgE-mediated. Non-IgE-mediated TNs allergy is less frequent, especially in children (21). A high rate of co-sensitization and co-allergy to other TNs and to peanut is observed among different populations $(16,18,20,21)$. In this sample, about one-third of children had peanut sensitization and more than four fifths were sensitized to other TNs. The high homology between their proteins can explain this strong association. We stress out that these children were considered to be included in the high-risk group since they had personal or family history of allergy.

The diagnosis of TNs allergy is made by the combination of a typical clinical presentation and evidence of TNs sensitization, assessed by in vivo tests (skin prick tests) or in vitro tests (identification of specific IgE antibodies in the serum) $(7,16,18)$. Skin prick test equal to or greater than $8 \mathrm{~mm}$ or specific IgE test equal to or greater than $15 \mathrm{kU} / \mathrm{L}$ is highly predictive of clinical allergy, although do not predict its severity $(16,18)$. Double-blind, placebo-controlled oral food challenges are the gold standard for the diagnosis of food allergy $(7,16)$. They are usually not necessary in TNs allergy but may be used to confirm or refute the diagnosis, when history and test results are conflicting $(7,16,18)$. In our study no oral challenge test has been performed since all included children had a severe systemic reaction clearly related to the TN ingestion and had a proven sensitization to the culprit TN.

As reported by other authors $(24,25)$, less than half of children admitted to the emergency department during the anaphylactic episode were treated with epinephrine. Intramuscular epinephrine injection in the anterolateral thigh is the first-line treatment for anaphylaxis and should be administered as soon as possible $(7,12,26)$. It is the only effective drug to prevent progression of the anaphylactic reaction and its delayed administration is con- sidered a risk factor for a fatal outcome $(3,7,16)$. The underuse of epinephrine by healthcare professionals can be explained by the complexity involved in establishing the diagnosis of anaphylaxis, lack of knowledge of how to administer epinephrine and use epinephrine auto-injectors, and misconceptions about epinephrine safety $(15,25)$. To help improving the appropriate use of epinephrine in patients diagnosed with anaphylaxis within the emergency setting, physician training programs should be implemented.

Anaphylaxis may be even more difficult to recognize and diagnose in younger children, mainly in infants, due to inherent differences in their ability to communicate their symptoms $(12,23,27,28)$. This difficulty may also be explained by the subjectivity of the clinical symptoms such as abdominal cramps, sudden cry and irritability, common in this age group, where frequently occurs the first clinical manifestation of the sensitization to the allergen $(12,23,27)$.

An epinephrine auto-injector was prescribed to all children at the allergy outpatient department after the first TN induced anaphylactic event. As found in the literature, the prescription of epinephrine auto-injectors is strongly recommended for the proper management of future occurrence of anaphylaxis $(3,16,18)$. Furthermore, encouraging these patients and caregivers to carry the auto-injectors all the time is an essential part of training (18).

TNs allergy can be severe, and usually persists over time, although resolution has been documented in some children (18). Although it was initially believed that TNs allergy rarely resolves, subsequent studies have shown that tolerance can develop in a minority of patients over time (up to $9 \%)(3,18,20)$. The predictors of outgrowing TNs allergies are a low or undetectable specific IgE levels, absence of other food/TNs allergy and a history of outgrowing peanut allergy (3).

The pillars of food allergy management are strict avoidance of the culprit allergens, prompt treatment of symptoms upon accidental exposure, patient and caregiver education (including food allergen labeling, food preparation and the risks of occult exposure), and management of allergic comorbidities $(3,16,18)$. Allergen avoidance diets should be specific and limited to the relevant foods to minimize both risks of an allergic reaction and over-restriction $(9,20)$. Complete nut avoidance is the safest approach, reducing the risk of an accidental reaction. This recommendation aims to simplify the message and improve avoidance while eating in schools and restaurants. However, it is difficult to achieve and can result in a significant restriction of certain food products. There are also nutritional, cultural and immunological arguments for the allowance consumption of other nuts (18). If a patient is already consuming a nut that he is not allergic to, it is reasonable to continue consuming it on a regular basis $(18,20)$. The decision to avoid all nuts (all nut exclusion) or only the culprit nut (single nut exclusion) should ultimately involve the 
patient and his family (16). Although in adults it is safe to avoid specifically the culprit nut in the patient's diet, at pediatric age, the decision to avoid all nuts is more frequent, mainly at school. Furthermore, within a restaurant environment, all nut exclusion diet is always the safest approach, due to the risk of misidentification or inadvertent substitution with other nut types.

To our knowledge, this is the first Portuguese study about TNs-induced anaphylactic reaction in a preschool age population. However, the authors findings might not be directly applicable to other populations due to geographical and cultural differences.

\section{References}

1. Sicherer SH, Sampson HA. Food allergy: epidemiology, pathogenesis, diagnosis, and treatment. J Allergy Clin Immunol 2014; 133(2):291-307.

2. Umasunthar T, Leonardi-Bee J, Turner PJ, Hodes M, Gore C, Warner $\mathrm{JO}$, et al. Incidence of food anaphylaxis in people with food allergy: A systematic review and meta-analysis. Clin Exp Allergy 2015; 45(11):1621-36.

3. Lee S. IgE-mediated food allergies in children: prevalence, triggers, and management. Korean J Pediatr 2017; 60(4):99-105.

4. Sicherer SH, Sampson HA. Food allergy: A review and update on epidemiology, pathogenesis, diagnosis, prevention, and management. J Allergy Clin Immunol 2018; 141(1):41-58.

5. Liew WK, Williamson E, Tang ML. Anaphylaxis fatalities and admissions in Australia. J Allergy Clin Immunol 2009; 123(2):434-42.

6. Panesar SS, Javad S, de Silva D, Nwaru BI, Hickstein L, Muraro A, et al. EAACI Food Allergy and Anaphylaxis Group. The epidemiology of anaphylaxis in Europe: a systematic review. Allergy 2013; 68(11):1353-61.

7. Muraro A, Roberts G, Worm M, Bilò MB, Brockow K, Fernández Rivas M, et al. Anaphylaxis: guidelines from the European Academy of Allergy and Clinical Immunology. Allergy 2014; 69(8):1026-45.

8. Motosue MS, Bellolio MF, Van Houten HK, Shah ND, Campbell RL. National trends in emergency department visits and hospitalizations for food-induced anaphylaxis in US children. Pediatr Allergy Immunol 2018; 29(5):538-44.

9. Braganza S, Acworth J, Mckinnon D, Peake JE, Brown AF. Paediatric emergency department anaphylaxis: different patterns from adults. Arch Dis Child 2006; 91(2):159-63.

10. Morais-Almeida M, Gaspar A, Santa-Marta C, Piedade S, Leiria-Pinto P, Pires G, et al. Anaphylaxis: from notification and knowledge to management. Rev Port Imunoalergologia 2007; 15(1):19-41.

11. Silva R, Gomes E, Cunha L, Falcao H. Anaphylaxis in children: a nine years retrospective study (2001-2009). Allergol Immunopathol (Madr) 2012; 40(1):31-6.

12. Silva D, Gaspar Â, Couto M, Morais-Almeida M. Anaphylaxis at pediatric age: From infancy to adolescence. Rev Port Imunoalergologia 2013; 21(3):157-75.

13. Gaspar Â, Santos N, Piedade S, Santa-Marta C, Pires G, Sampaio $\mathrm{G}$, et al. One-year survey of paediatric anaphylaxis in an allergy department. Eur Ann Allergy Clin Immunol 2015; 47(6):197-205.

14. Johnson J, Malinovschi A, Alving K, Lidholm J, Borres MP, Nordvall L. Ten-year review reveals changing trends and severity of allergic reactions to nuts and other foods. Acta Paediatr 2014; 103(8):862-7.
In conclusion, potentially life-threatening TNs allergy may occur early in childhood and adequate management should be undertaken. There is a need for further studies to better characterize TNs-induced anaphylaxis prevalence and clinical manifestations in the young children population, particularly in Europe.

\section{Conflict of interests}

The authors declare that they have no conflict of interests.

15. Jeong K, Lee SY, Ahn K, Kim J, Lee HR, Suh DI, et al. A multicenter study on anaphylaxis caused by peanut, tree nuts, and seeds in children and adolescents. Allergy 2017; 72(3):507-10.

16. Weinberger T, Sicherer S. Current perspectives on tree nut allergy: a review. J Asthma Allergy 2018; 11:41-51.

17. McWilliam V, Koplin J, Lodge C, Tang M, Dharmage S, Allen K. The Prevalence of Tree Nut Allergy: A Systematic Review. Curr Allergy Asthma Rep 2015; 15(9):54.

18. Stiefel G, Anagnostou K, Boyle RJ, Brathwaite N, Ewan P, Fox AT, et al. BSACI guideline for the diagnosis and management of peanut and tree nut allergy. Clin Exp Allergy 2017; 47(6):719-39.

19. Fernandes RA, Regateiro F, Pereira C, Faria E, Pita J, Todo-Bom A, et al. Anaphylaxis in a food allergy outpatient department: oneyear review. Eur Ann Allergy Clin Immunol 2018; 50(2):81-8.

20. Muraro A, Werfel T, Hoffmann-Sommergruber K, Roberts G, Beyer K, Bindslev-Jensen C, et al. EAACI food allergy and anaphylaxis guidelines: diagnosis and management of food allergy. Allergy 2014; 69(8):1008-25.

21. Liu M, Burks AW, Green TD. Tree nut allergy: risk factors for development, mitigation of reaction risk and current efforts in desensitization. Expert Rev Clin Immunol 2015; 11(5):673-9.

22. Sampson HA, Muñoz-Furlong A, Campbell RL, Adkinson NF Jr, Bock SA, Branum A, et al. Second symposium on the definition and management of anaphylaxis: summary report - Second National Institute of Allergy and Infectious Disease/Food Allergy and Anaphylaxis Network symposium. J Allergy Clin Immunol 2006; 117(2):391-7.

23. Rudders SA, Banerji A, Clark S, Camargo CA Jr. Age-related differences in the clinical presentation of food-induced anaphylaxis. J Pediatr 2011; 158(2):326-8.

24. Solé D, Ivancevich JC, Borges MS, Coelho MA, Rosário NA, Ardusso L, et al. Latin American Anaphylaxis Working Group. Anaphylaxis in Latin American children and adolescents: the Online Latin American Survey on Anaphylaxis (OLASA). Allergol Immunopathol (Madr) 2012; 40(6):331-5.

25. Prince BT, Mikhail I, Stukus DR. Underuse of epinephrine for the treatment of anaphylaxis: missed opportunities. J Asthma Allergy 2018; 11:143-51.

26. Carneiro-Leão L, Santos N, Gaspar Â. Anaphylaxis, Diagnosis and Treatment. Acta Med Port 2018; 31(2):134-5.

27. Simons FE. Anaphylaxis in infants: can recognition and management be improved? J Allergy Clin Immunol 2007; 120(3):537-40.

28. Dosanjh A. Infant anaphylaxis: the importance of early recognition. J Asthma Allergy 2013; 6:103-7. 